\title{
44583 - PARENTS IN THE POST-ANESTHETIC RECOVERY ROOM: WHO BENEFITS MOST?
}

\author{
Bruce Dick, University of Alberta, Edmonton, AB, Canada; \\ David Lardner, Alberta Children's Hospital; \\ Susan Crawford, Alberta Children's Hospital;
}

INTRODUCTION: Minimizing the separation of parent and child in hospital has been a philosophy of paediatric care for some time. Some children's hospitals have a long history of parental presence in the Post-Anesthetic Recovery Room (PARR) while others do not include this as routine practice. There is a small but growing literature on the effect of parental presence in the PARR. This study was conducted in order to further examine the effects of parental presence in the PARR on children's pain, anxiety, and other postoperative behavioural measures. METHODS: Local IRB approval was obtained for this study. 300 families of children (aged 2-8 years) scheduled for elective day surgery whose PARR stay was anticipated to be $>10$ minutes were recruited. Participants were randomly assigned to parental presence or no parental presence in the PARR. Demographic, anaesthetic, and analgesic information was recorded. Data were also gathered for postoperative pain (CHEOPS), preoperative anxiety (m-YPAS), emergence agitation and distress, and postoperative behavioural change (PHBQ) two weeks after surgery. RESULTS: We have reported elsewhere that children less than 5-years of age, those who had more painful procedures, those with higher CHEOPS scores after leaving the PARR, and those whose parents were not present in the PARR were more likely to be reported to have significantly more behavioural change following surgery. Children below the age of 5-years were observed to show more agitation and crying behaviours in the PARR than older children. We also found that children less than 5-years of age who were not involved in a surgery preparation class were found to exhibit significantly more crying/inconsolable/severely restless behaviour than those who had attended such a class. No differences were found on this measure between older children who had or had not attended such a class. Further, children younger than 5-years who had a parent present during anesthesia induction were found to show significantly more crying/inconsolable/severely restless behaviour if their parent was absent from the PARR. SUMMARY: There appear to be many factors that play a role in children's emotional and behavioural responses before and after surgery. Our findings suggest that developmental level, postoperative pain, and parental presence in the PARR may have a significant impact on children's behaviour well after a child leaves the hospital. In addition, other factors such as whether or not children receive pre-operative preparation for surgery and whether or not their parents are present during anesthetic induction can also play an important role in their behavioural responses following surgery. 